\title{
Systematic studies of the molecular properties of intrashell and intershell doubly excited states of $\mathrm{C}^{4+}$ and $\mathrm{He}$
}

\author{
M Dunn, Z Chen and C D Lin \\ Department of Physics, Kansas State University, Manhattan, KS 66506-2601, USA \\ Received 26 September 1989 , in final form 16 February 1990
}

\begin{abstract}
The molecular (and antimolecular) properties of a large number of intrashell and intershell doubly excited states of $\mathrm{C}^{4+}$ and $\mathrm{He}$ are studied. By comparing a number of expectation values using wavefunctions calculated by the truncated diagonalisation method (TDM) and the group theoretic doubly excited symmetry basis (DESB) functions, the origin of the molecular properties of doubly excited states are examined. In particular, properties such as rotor structure, rotational contraction and $T$-doubling are inherent in the DESB approximation where the rotational energy separation is due to the bielectronic repulsion. In the full TDM calculation, it is shown that whenever intershell coupling is strong, such as in $\mathrm{He}$, a molecule-like rotational expansion along a rotor series becomes possible. An extensive calculation of momentum correlation, $\left\langle\boldsymbol{p}_{1} \cdot \boldsymbol{p}_{2}\right\rangle$, has also been carried out for many intrashell and intershell doubly excited states and the results are shown to be generally consistent with the classical molecular model of Krause, Morgan and Berry.
\end{abstract}

\section{Introduction}

The observation of the anomalous absorption spectra of doubly excited He by Madden and Codling (1963) was immediately recognised by Fano and co-workers (Cooper et al 1963) to imply strongly correlated electron motion in these states. Since then our knowledge about these states has improved considerably and several methods have been devised which point towards an understanding of this strong correlation. The adiabatic hyperspherical approach (Macek 1968, Lin 1983, 1984, 1986, Watanabe and Lin 1986) identifies the hyperradius $R \equiv\left(r_{1}^{2}+r_{2}^{2}\right)^{1 / 2}$ as a slow variable and so solves for a system of the helium isoelectronic sequence in a fashion similar to the BornOppenheimer approximation. The Born-Oppenheimer approximation itself (Feagin 1987, Feagin and Briggs 1987, 1988) has been used with success, treating the interelectron separation $r_{12}$ as the slow variable. Doubly excited states have also been approached via a dimensional scaling treatment (Herschbach et al 1988).

Herrick and Sinanoğlu (Herrick and Sinanoğlu 1975, Sinanoğlu and Herrick $1975 \mathrm{a}, \mathrm{b}$, also see Wulfman 1973) studied the breaking of the bound state Coulombic $S O(4) \times S O(4)$ dynamical symmetry of hydrogenic states by the inter-electron interaction $1 / r_{12}$. They define a set of basis states which transform under an $S O(4)$ subgroup of $S O(4) \times S O(4)$ and are labelled by the new quantum numbers $K$ and $T$ replacing the more familiar angular momentum of the individual electrons as used in the independent electron approximation. Herrick and Sinanoğlu call their symmetry basis a doubly excited symmetry basis (DESB). These authors found that if the full twoelectron wavefunction is expanded in the complete set of DESB states, the wavefunctions are dominated by DESB states with the same values of $K$ and $T$. In other words, $K$ 
and $T$ are approximate quantum numbers for the two-electron system and can be used to label the states. We shall adopt the notation for classifying states ${ }_{n}(K, T)_{N}$ where $n$ and $N$ are the principal quantum numbers of the outer and inner electrons respectively (Lin 1984, 1986).

Herrick et al (Herrick and Kellman 1980, Herrick et al 1980, Kellman and Herrick 1980, Herrick 1983) subsequently recognised a supermultiplet structure for doubly excited intrashell $(n=N)$ states from which an approximate rovibrator energy level pattern for a linear triatomic molecule could be delineated. The correspondence between the atomic quantum numbers and the molecular quantum numbers was found to be

$$
\begin{aligned}
& K \leftrightarrow N-\nu-1 \\
& T \leftrightarrow l_{\mathrm{v}}
\end{aligned}
$$

where $l_{\mathrm{v}}$ is the vibrational angular momentum, $\nu$ is the vibrational (i.e. bending) quantum number. Herrick and Kellman (1980) speculated that intershell (e.g. $2 l 3 l^{\prime}$ ) states may exhibit the stretching modes of a triatomic linear molecule. Subsequently Berry and co-workers (Berry and Krause 1988, Berry 1989) used well converged configuration interaction $(\mathrm{CI})$ wavefunctions from a Sturmian basis to demonstrate the existence of molecular (rovibrator) correlations for the $2 l 2 l^{\prime}$ states and found that indeed the intershell $2 \mathrm{~s} 3 \mathrm{~s}$ states displayed molecule-like symmetric and antisymmetric stretch modes. Lin $(1983,1984)$ has introduced an empirical radial quantum number $A=0, \pm 1$ where $A=+1$ states exhibit symmetric stretch, $A=-1$ states exhibit antisymmetric stretch, and $A=0$ states for which the radial correlation properties resemble those of singly excited states. The quantum number $A$ is not an independent label and is completely determined by the other quantum numbers labelling the state. A given Rydberg series (or channel in the hyperspherical parlance) will be denoted by the approximate quantum numbers $(K, T)_{N}^{A}$. Watanabe and Lin (1986) have explored the connections between the molecular picture and the hyperspherical approach. It should also be noted that the Born-Oppenheimer method invokes quantum numbers similar to the $K, T$ quantum numbers (Feagin and Briggs 1987, Feagin 1987, Feagin and Briggs 1988).

Krause et al (1987) subsequently demonstrated that the molecular picture is capable of some quantitative prediction. Classically one would expect the sign of $p_{1} \cdot p_{2}$, where $p_{i}$ is the momentum of the $i$ th electron, to depend on the nature of the molecular excitation. For the vibration and antisymmetric stretch modes $\boldsymbol{p}_{1} \cdot \boldsymbol{p}_{2}$ will be positive, while for the rotor and symmetric stretch modes $\boldsymbol{p}_{1} \cdot \boldsymbol{p}_{2}$ will be negative. Krause et al find that the well converged $C$ I wavefunctions for the $n=N=2$ manifold and $2 \mathrm{~s} 3 \mathrm{~s}{ }^{1,3} \mathrm{~S}^{\mathrm{e}}$ states do display this correlation. One should caution that the number of states studied is small and that a classical picture may not be appropriate for states of such low modes of excitation. Mølmer and Taulbjerg (1988) and Iwai and Nakamura (1989a, b) have elucidated much of the physics behind the molecular behaviour of doubly excited states and Chen and Lin (1989) have recently extended the classification scheme to include systems of three particles with arbitrary masses.

Despite the notable successes of the molecular picture, $A= \pm 1$ doubly excited states exhibit many 'antimolecular' properties. In particular the abrupt 'cut-off' of the various rotor series about some angular momentum and the tendency of $\left\langle\cos \theta_{12}\right\rangle$ to take on larger values as the angular momentum increases up a rotor series. The rotational constant $B$ is roughly four times too small to result from the moment of inertia and Watanabe and Lin (1986) have speculated that $B$ derives from the interelectronic 
repulsion which mocks the purely inertial energy level structure of a rotor: This suggestion was based on a simple study of the behaviour of intrashell DESB states. In addition $B$ tends to increase with angular momentum, whereas for a molecule it decreases. As a final example, although the list is far from complete, doubly excited states are understood to contract with increasing angular momentum. These properties are largely adduced from studies of DESB states and hyperspherical potential curves.

In the present paper a study is made of the isoelectronic $\mathrm{C}^{4+}$ and He systems with the aim of investigating a much larger set of states than was previously considered by Lin and co-workers (Lin 1983, 1984, 1986, Watanabe and Lin 1986) or Berry and co-workers (Krause et al 1987, Berry and Krause 1988, Berry 1989) although the study is not as ambitious for the individual states concerned.

Calculational procedures and classification methods used are discussed in section 2 and the details of the study are presented in sections 3 to 7 . Section 8 recaps and provides an overview of the major results and conclusions to be drawn from this study.

\section{Methods and classification procedures}

\subsection{Calculation and analysis of wavefunctions}

The resonance wavefunctions are generated by the truncated diagonalisation method (TDM) (Lipsky et al 1977) which is a cl method with a truncated hydrogenic basis set. The basis set excludes all singly excited and continum states and so the derived energies are upper bounds to the eigenvalues of QHQ (Lipsky et al 1977) where $H$ is the Hamiltonian of the two-electron system and $Q$ is the Feshbach projection operator projecting out the states excluded from the basis set. Results for the energies compare favourably with calculation of Lipsky et al (1977) and are frequently better due to the procedure of adopting selective basis functions in the present study. Hydrogenic states with $n$ and $l$ up to 14 and 6 respectively are included in the wavefunction for He. The resonance energies and wavefunctions will not be as accurate as those obtained from a Sturmian basis or the complex-coordinate rotation method (Junker 1982, Reinhardt 1982, Ho 1983, 1987, 1989) but are adequate for the current task. The 'raw data' for this study are the $N=3$ Rydberg series of $\mathrm{C}^{4+}$ and the $N=2$ and 3 Rydberg series of He although this paper will not explicitly discuss the $N=2$ series of He. All of the conclusions from the $N=3$ series apply to the $N=2$ series.

Berry et al (Berry and Krause 1988, Berry 1989) study conditional probability distributions and $\operatorname{Lin}(1983,1984,1986)$ studies the surface charge densities of the adiabatic hyperspherical basis functions and the adiabatic hyperradial potential curves. The current paper is more modest and searches for regular behaviour of a number of expectation values, namely $\left\langle\cos \theta_{12}\right\rangle,\langle\mathrm{KE}\rangle=\frac{1}{2}\left\langle\boldsymbol{p}_{1}^{2}+\boldsymbol{p}_{2}^{2}\right\rangle,\left\langle\boldsymbol{p}_{1} \cdot \boldsymbol{p}_{2}\right\rangle,\left\langle 1 / \boldsymbol{r}_{12}\right\rangle$ and $\overline{\boldsymbol{R}}^{2}=\left\langle\boldsymbol{R}^{2}\right\rangle$, in addition to the resonance energy $E$ (the approach adopted in this paper for calculating $\left\langle\boldsymbol{p}_{1} \cdot \boldsymbol{p}_{2}\right\rangle$ differs from Krause $e t$ al (1987) and is outlined in the appendix). Such regular behaviour usually enables one to classify the states according to the $(K, T)$ quantum numbers but in cases of ambiguity the wavefunctions are projected onto the DESB states. Throughout this paper the linear triatomic molecular model is used as a point of reference to which the behaviour of the above expectation values are referred. If doubly excited states were genuinely molecular then as. $L$ increases $E$ would display a rotor structure, $\left\langle\cos \theta_{12}\right\rangle$ would decrease, $\langle K E\rangle$ would increase and $\bar{R}^{2}$ would increase. The behaviour of $\left\langle\boldsymbol{p}_{1} \cdot \boldsymbol{p}_{2}\right\rangle$, as stated earlier, would be positive for vibration and antisymmetric stretch modes, and negative for the rotor and symmetric stretch modes. 


\subsection{The calculation of $\left\langle\boldsymbol{p}_{1} \cdot \boldsymbol{p}_{2}\right\rangle$}

The accuracy of the calculated momentum correlation, $\left\langle\boldsymbol{p}_{1} \cdot \boldsymbol{p}_{2}\right\rangle$, needs some discussion. One notes that for all intrashell states and some intershell states the terms one would expect to be dominant in the expression for $\left\langle\boldsymbol{p}_{1} \cdot \boldsymbol{p}_{2}\right\rangle$, those deriving from the dominant terms in the wavefunction, are zero. This follows from two facts: (i) the matrix elements and hence reduced matrix elements of $p$ are zero unless the angular momentum of the two states differ by unity, (ii) all intrashell hydrogenic matrix elements of $p$, and hence all intrashell hydrogenic reduced matrix elements of $p$, are zero. This latter point may be understood as follows. Clearly all hydrogenic bound state matrix elements of $p$ which are diagonal in $n$ and $l$ are zero, but due to the bound state Coulombic $S O(4)$ dynamical symmetry any linear combination of states with the same principal quantum number will be a bound state eigenfunction. Hence $\langle\boldsymbol{p}\rangle$ is zero between these new hybrid states. In particular one may form the linear combinations $\left.(|n| m\rangle+\left|n l^{\prime} m\right\rangle\right)$ and $(|n l m\rangle-|n l m\rangle)$ for which $\langle\boldsymbol{p}\rangle$ in both of these states are zero. This demands that $\left\langle n l^{\prime} m|\boldsymbol{p}| n l m\right\rangle=0$ which in turn implies that the reduced matrix element $\left\langle n l^{\prime}|| \boldsymbol{p}|| n l\right\rangle=0$.

This means that the non-zero value of $\left\langle\boldsymbol{p}_{1} \cdot \boldsymbol{p}_{2}\right\rangle$ for intrashell states is generated by terms deriving from the poorly converged tail of the expansion for the wavefunction, so less than accurate results are to be expected. The authors believe that for some states of $\mathrm{He}$ the quoted values for $\left\langle\boldsymbol{p}_{1} \cdot \boldsymbol{p}_{2}\right\rangle /\left(m_{\mathrm{e}}\langle\mathrm{KE}\rangle\right)$ may be accurate to no more than $10^{-2}$, although for other states, particularly intershell states, the quoted result will be more accurate. The authors generally limit themselves to drawing conclusions about the sign of $\left\langle\boldsymbol{p}_{1} \cdot \boldsymbol{p}_{2}\right\rangle$ although in many cases more precise inferences may be drawn from the data.

\section{Systematics of static properties of doubly excited states}

\subsection{General features of the $(2,0)_{3}^{+}$rotor series}

3.1.1. The $C^{4+}$ system. Table 1 lists the energy and a number of expectation values of the intrashell $(2,0)_{3}^{+}$states for $\mathrm{C}^{4+}$ from the present TDM calculation and from the approximate DESB wavefunctions (shown in parentheses).

Any difference between the TDM and the DESB originates from the inclusion of intershell states in the former wavefunction. There are a number of regularities displayed in this table which are characteristic of doubly excited states: (i) the energy levels show the approximate rotor-like structure of a molecule; (ii) the average of $\cos \theta_{12}$

Table 1. Tabulation of the energy and various expectation values for the intrashell ${ }_{3}(2,0)_{3}^{+}$ rotor series of $\mathrm{C}^{4+}$ (DESB values in brackets). All intrashell DESB values of $\left\langle p_{1} \cdot p_{2}\right\rangle / m_{e}$ are zero and are thus not tabulated.

\begin{tabular}{llllll}
\hline & $E(\mathrm{eV})$ & $\left\langle\cos \theta_{12}\right\rangle$ & $\bar{R}^{2}(\mathrm{au})^{2}$ & $\left\langle 1 / r_{12}\right\rangle(\mathrm{eV})$ & $\begin{array}{l}\left\langle\boldsymbol{p}_{1} \cdot \boldsymbol{p}_{2}\right\rangle / \boldsymbol{m}_{\mathrm{e}} \\
(\mathrm{eV})^{\prime}\end{array}$ \\
\hline${ }^{1} \mathrm{~S}^{\mathrm{e}}$ & $-101.04(-100.79)$ & $-0.558(-0.556)$ & $11.70(10.75)$ & $7.57(8.05)$ & -0.25 \\
${ }^{3} \mathrm{P}^{\circ}$ & $-100.77(-100.53)$ & $-0.513(-0.500)$ & $11.30(10.38)$ & $7.80(8.32)$ & -0.21 \\
${ }^{1} \mathrm{D}^{\mathrm{e}}$ & $-100.00(-99.65)$ & $-0.424(-0.389)$ & $10.67(9.63)$ & $8.46(9.20)$ & -0.27 \\
${ }^{3} \mathrm{~F}^{\mathrm{o}}$ & $-98.73(-98.23)$ & $-0.286(-0.222)$ & $9.63(8.5)$ & $9.57(10.62)$ & -0.19 \\
${ }^{1} \mathrm{G}^{\mathrm{e}}$ & $-95.42(-94.18)$ & $-0.126(-0.000)$ & $8.48(7.0)$ & $12.09(14.67)$ & -0.44 \\
\hline
\end{tabular}


increases with $L$; (iii) the Coulomb repulsion energy $\left\langle 1 / r_{12}\right\rangle$ increases with $L$; (iv) the average size of each atomic state, measured by $\bar{R}^{2}$, decreases with $L$. One also notes that for this ${ }_{3}(2,0)_{3}^{+}$series, $\left\langle\boldsymbol{p}_{1} \cdot \boldsymbol{p}_{2}\right\rangle$ is negative.

In comparing the TDM and the DESB functions, the inclusion of intershell states in the basis function lowers the energy of each doubly excited state, increases the angle $\theta_{12}$ between the two electrons and makes the size of each state larger by decreasing the value of $\left\langle 1 / \boldsymbol{r}_{12}\right\rangle$. The intershell coupling is also responsible for the non-zero $\left\langle\boldsymbol{p}_{1} \cdot \boldsymbol{p}_{2}\right\rangle$ for each intrashell state.

From this table two features of molecular behaviour stand out clearly: (i) the energy levels indeed show approximate rotor-like structure; (ii) $\left\langle\boldsymbol{p}_{1} \cdot \boldsymbol{p}_{2}\right\rangle$ is negative in accordance with the expected value of a classical linear triatomic molecule with zero vibrational quantum number and no stretch excitation (Krause et al 1987). Note that $\left\langle\boldsymbol{p}_{1} \cdot \boldsymbol{p}_{2}\right\rangle$ is zero according to the DESB approach, so this aspect of the molecular character is not built into this approximation.

This table also points out a number of aspects which are not characteristic of a molecule. (i) The rotor structure has a non-inertial origin; the bielectronic repulsion accounts for about $85 \%$ of the increase in energy between one member of the rotor series and the next. This is consistent with the suggestion of Watanabe and Lin (1986). (ii) $\left\langle\cos \theta_{12}\right\rangle$ displays an antimolecular rise with increasing $L$. (iii) The centrifugal contraction, as measured by the decrease of $\bar{R}^{2}$ with $L$. Due to the centrifugal distortion, a molecular model would give larger value of $\bar{R}^{2}$ for larger $L$.

3.1.2. The He system. In table 2 we tabulate similar expectation values for the same intrashell series for helium. The general trend for each expectation value along the rotor series is similar to that for $\mathrm{C}^{4+}$. However, the relatively more important bielectronic repulsion opens the angle $\theta_{12}$ somewhat. Values of $\bar{R}^{2}$ fall as $L$ increases, but the last member, ${ }^{1} \mathrm{G}^{\mathrm{e}}$, shows an expansion on the previous member, ${ }^{3} \mathrm{~F}^{\circ}$. Values of $\left\langle 1 / r_{12}\right\rangle$ rise with increasing $L$, but the rise is only sufficient to account for some $40 \%$ of the rise of the total energy $E$ up to a rotor series. We also show the expectation values of the kinetic energy operator which decrease with increasing $L$-contrary to the trend of a molecular rotor.

Table 2. Tabulation of the energy and various expectation values for the intrashell ${ }_{3}(2,0)_{3}^{+}$ rotor series of $\mathrm{He}$.

\begin{tabular}{lllllll}
\hline & $E(\mathrm{eV})$ & $\left\langle\cos \theta_{12}\right\rangle$ & $\bar{R}^{2}(\mathrm{au})^{2}$ & $\begin{array}{l}\left\langle 1 / \boldsymbol{r}_{12}\right\rangle \\
(\mathrm{eV})\end{array}$ & $\begin{array}{l}\left\langle\boldsymbol{p}_{1} \cdot \boldsymbol{p}_{2}\right\rangle /\left(m_{\mathrm{e}}\langle\mathrm{KE}\rangle\right) \\
\times 10^{-2}\end{array}$ & $\langle\mathrm{KE}\rangle(\mathrm{eV})$ \\
\hline${ }^{1} \mathrm{~S}^{\mathrm{e}}$ & -9.63 & -0.605 & 123.5 & 2.27 & -0.466 & 8.77 \\
${ }^{3} \mathrm{P}^{\mathrm{o}}$ & -9.56 & -0.572 & 120.7 & 2.32 & -0.572 & 8.75 \\
${ }^{1} \mathrm{D}^{\mathrm{e}}$ & -9.36 & -0.511 & 117.8 & 2.43 & -1.16 & 8.45 \\
${ }^{3} \mathrm{~F}^{\mathrm{o}}$ & -9.04 & -0.421 & 112.0 & 2.60 & -2.47 & 8.31 \\
${ }^{1} \mathrm{G}^{\mathrm{e}}$ & -8.38 & -0.347 & 113.5 & 2.76 & -7.94 & 7.96 \\
\hline
\end{tabular}

3.1.3. Comparison of $A=+1$ intrashell and intershell series. In this section we compare the expectation values of the intrashell and intershell states which have identical $K$, $T$ and $A$ quantum numbers. Results are shown in table 3 where the ${ }_{3}(2,0)_{3}^{+}$and ${ }_{4}(2,0)_{3}^{+}$ series are compared, the $n=4$ series having one quantum of symmetric stretch. A 
Table 3. Comparison of the expectation values $\left\langle\cos \theta_{12}\right\rangle,\left\langle 1 / r_{12}\right\rangle$ and $\bar{R}$ for the intrashell ${ }_{3}(2,0)_{3}^{+}$first intershell ${ }_{4}(2,0)_{3}^{+}$and ${ }_{4}(2,0)_{3}^{-}$rotor series of $C^{4+}$ (DESB values in brackets).

\begin{tabular}{|c|c|c|c|c|}
\hline & \multicolumn{3}{|c|}{$E(\mathrm{eV})$} & \\
\hline & \multicolumn{2}{|c|}{$(2,0)^{+}$} & \multirow[t]{2}{*}{$(2,0)^{-}$} & \\
\hline & intra & inter & & \\
\hline${ }^{1} S^{e}$ & $-101.04(-100.79)$ & $-79.17(-78.92)$ & $-79.92(-79.82)$ & ${ }^{3} \mathrm{~S}^{\mathrm{e}}$ \\
\hline${ }^{3} \mathrm{P}^{0}$ & $-100.77(-100.53)$ & $-79.08(-78.80)$ & $-79.79(-79.66)$ & ${ }^{1} \mathrm{P}^{\circ}$ \\
\hline${ }^{1} D^{e}$ & $-100.00(-99.65)$ & $-78.57(-78.30)$ & $-79.48(-79.30)$ & ${ }^{3} \mathrm{D}^{\mathrm{e}}$ \\
\hline${ }^{3} \mathrm{~F}^{\circ}$ & $-98.73(-98.23)$ & $-77.96(-77.66)$ & $-78.89(-78.59)$ & ${ }^{1} \mathrm{~F}^{\circ}$ \\
\hline \multirow[t]{4}{*}{$\mathrm{G}^{\mathrm{e}}$} & $-95.42(-94.18)$ & $-76.66(-76.27)$ & $-77.75(-77.20)$ & ${ }^{3} G^{e}$ \\
\hline & \multicolumn{3}{|c|}{$\left\langle\cos \theta_{12}\right\rangle$} & \\
\hline & \multicolumn{2}{|c|}{$(2,0)^{+}$} & \multirow[t]{2}{*}{$(2,0)^{-}$} & \\
\hline & intra & inter & & \\
\hline${ }^{1} S^{e}$ & $-0.558(-0.556)$ & $-0.581(-0.592)$ & $-0.610(-0.608)$ & ${ }^{3} S^{e}$ \\
\hline${ }^{3} \mathrm{P}^{0}$ & $-0.513(-0.500)$ & $-0.554(-0.557)$ & $-0.565(-0.563)$ & ${ }^{1} \mathrm{P}^{\circ}$ \\
\hline${ }^{1} \mathrm{D}^{\mathrm{e}}$ & $-0.424(-0.389)$ & $-0.504(-0.491)$ & $-0.474(-0.468)$ & ${ }^{3} \mathrm{D}^{\mathrm{e}}$ \\
\hline${ }^{3} F^{\circ}$ & $-0.286(-0.222)$ & $-0.409(-0.404)$ & $-0.336(-0.316)$ & ${ }^{1} \mathrm{~F}^{\circ}$ \\
\hline \multirow[t]{4}{*}{${ }^{1} G^{e}$} & $-0.126(-0.000)$ & $-0.335(-0.311)$ & $-0.168(-0.089)$ & ${ }^{3} \mathrm{G}^{\mathrm{e}}$ \\
\hline & \multicolumn{3}{|c|}{$\left\langle 1 / r_{12}\right\rangle(\mathrm{eV})$} & \\
\hline & \multicolumn{2}{|c|}{$(2,0)^{+}$} & \multirow[t]{2}{*}{$(2,0)^{-}$} & \\
\hline & intra & inter & & \\
\hline${ }^{1} \mathbf{S}^{\mathbf{e}}$ & $7.57(8.05)$ & $5.62(6.12)$ & $4.99(5.21)$ & ${ }^{3} S^{e}$ \\
\hline${ }^{3} \mathrm{P}^{\circ}$ & $7.80(8.32)$ & $5.68(6.23)$ & $5.10(5.37)$ & ${ }^{1} \mathrm{P}^{\circ}$ \\
\hline${ }^{1} D^{e}$ & $8.46(9.20)$ & $6.12(6.73)$ & $5.36(5.73)$ & ${ }^{3} \mathrm{D}^{\mathrm{c}}$ \\
\hline${ }^{3} \mathrm{~F}^{\circ}$ & $9.57(10.62)$ & $6.68(7.38)$ & $5.87(6.44)$ & ${ }^{1} \mathrm{~F}^{\circ}$ \\
\hline \multirow[t]{4}{*}{${ }^{1} \mathrm{G}^{\mathrm{e}}$} & $12.09(14.67)$ & $7.75(8.77)$ & $6.75(7.83)$ & ${ }^{3} G^{e}$ \\
\hline & \multicolumn{3}{|c|}{$\bar{R}(\mathrm{au})$} & \\
\hline & \multicolumn{2}{|c|}{$(2,0)^{+}$} & \multirow[t]{2}{*}{$(2,0)^{-}$} & \\
\hline & intra & inter & & \\
\hline${ }^{1} S^{e}$ & $3.42(3.28)$ & $5.07(4.74)$ & $4.94(4.74)$ & ${ }^{3} S^{e}$ \\
\hline${ }^{3} p^{0}$ & $3.36(3.22)$ & $4.99(4.68)$ & $4.89(4.68)$ & ${ }^{1} \mathrm{P}^{\circ}$ \\
\hline${ }^{1} D^{e}$ & $3.27(3.10)$ & $4.94(4.55)$ & $4.80(4.55)$ & ${ }^{3} \mathrm{D}^{\mathrm{e}}$ \\
\hline${ }^{3} \mathrm{~F}^{\circ}$ & $3.10(2.92)$ & $4.78(4.35)$ & $4.68(4.35)$ & ${ }^{1} \mathrm{~F}^{\circ}$ \\
\hline \multirow[t]{4}{*}{${ }^{1} G^{e}$} & $2.91(2.65)$ & $4.61(4.06)$ & $4.52(4.06)$ & ${ }^{3} G^{e}$ \\
\hline & \multicolumn{3}{|c|}{$\left\langle\boldsymbol{p}_{1} \cdot \boldsymbol{p}_{2}\right\rangle / m_{\mathrm{e}}(\mathrm{eV})$} & \\
\hline & \multicolumn{2}{|c|}{$(2,0)^{+}$} & \multirow[t]{2}{*}{$(2,0)^{-}$} & \\
\hline & intra & inter & & \\
\hline${ }^{1} S^{e}$ & $-0.25(0.0)$ & $-4.21(-5.12)$ & $4.56(5.12)$ & ${ }^{3} S^{e}$ \\
\hline${ }^{3} \mathrm{P}^{0}$ & $-0.21(0.0)$ & $-5.26(-6.28)$ & $5.25(6.28)$ & ${ }^{1} \mathrm{p}^{\circ}$ \\
\hline${ }^{1} D^{c}$ & $-0.27(0.0)$ & $-6.12(-8.70)$ & $6.74(8.70)$ & ${ }^{3} \mathrm{D}^{\mathrm{e}}$ \\
\hline${ }^{3} \mathrm{~F}^{\circ}$ & $-0.19(0.0)$ & $-8.43(-12.57)$ & $8.98(12.57)$ & ${ }^{1} F^{\circ}$ \\
\hline${ }^{1} \mathrm{G}^{\mathrm{e}}$ & $-0.44(0.0)$ & $-11.09(-18.17)$ & $11.28(18.17)$ & ${ }^{3} G^{e}$ \\
\hline
\end{tabular}


comparison of the ${ }_{4}(2,0)_{3}^{+}$and ${ }_{4}(2,0)_{3}^{-}$series is left to section 3.2 . The general trends of the expectation values with respect to $L$ are similar for the intrashell and intershell states, both in the DESB and in the TDM calculations. One notes that the angle $\theta_{12}$ opens up more for intershell states. Values of $\left\langle\boldsymbol{p}_{1} \cdot \boldsymbol{p}_{2}\right\rangle$ are negative for all the states of both series, consistent with the classical picture. The absolute values of $\left\langle\boldsymbol{p}_{1} \cdot \boldsymbol{p}_{2}\right\rangle$ for the intershell states are much higher and reflects the behaviour of the 'zeroth order' intershell DESB values. Both TDM and DESB values of $\left\langle\boldsymbol{p}_{1} \cdot \boldsymbol{p}_{2}\right\rangle$ for the intershell states show a smooth increase toward more negative values with increasing $L$.

\subsection{Comparison between $A=+1$ and $A=-1$ intershell states}

3.2.1. General features. In this section the static properties of the $A=+1$ and $A=-1$ intershell states, having identical $K$ and $T$, are compared. For the $\mathrm{C}^{4+}$ system this is shown in table 3 . Like the $A=+1$ series, the energy levels of the $A=-1$ series display an approximate molecular rotor behaviour where the bielectronic repulsion accounts for most $(80 \%)$ of the rise in energy up the rotor series. All $A= \pm 13 l 4 l^{\prime}$ states of $C^{4+}$ show rotational contraction following the trend set by the 'zeroth order' DESB states. The DESB values for $\bar{R}$ are identical for both rotor series since the DESB wavefunctions consist of $3 l 4 l^{\prime}$ configurations only.

Any difference in $\bar{R}$ of the $A=+1$ or -1 states from the full TDM calculation is due to the relative strengths of intershell mixing for the two series. One may expect the bielectronic repulsion to be less for the $A=-1$ states as they exhibit an approximate nodal line when $r_{1}=r_{2}$ whereas $A=+1$ states exhibit an approximate antinodal line when $r_{1}=r_{2}$. From the above argument one expects $\left\langle 1 / r_{12}\right\rangle, E$ and $\bar{R}$ to be larger for the $A=+1$ states, and indeed, this is borne out in table 3 . Values of $\left\langle\cos \theta_{12}\right\rangle$ again follow the antimolecular trends of the DESB states, being more negative for low $L$ and larger for high $L$ members of the $A=-1$ series compared with the $A=+1$ series.

3.2.2. Symmetric stretch, antisymmetric stretch and $\left\langle\boldsymbol{p}_{1} \cdot \boldsymbol{p}_{2}\right\rangle$. The molecular model pictures the $A=+1$ states as symmetric stretch modes and the $A=-1$ states as antisymmetric stretch modes. Thus the value of $\left\langle\boldsymbol{p}_{1} \cdot \boldsymbol{p}_{2}\right\rangle$ assigns negative values for the $A=+1$ states and positive values for the $A=-1$ states. Table 3 shows this prediction is verified for $\mathrm{C}^{4+}$ and that the values are almost equal but opposite in sign for the two series. These results derive from the behaviour of $\left\langle\boldsymbol{p}_{1} \cdot \boldsymbol{p}_{2}\right\rangle$ for the $A= \pm 1$ 'zeroth order' DESB states and are exactly equal but opposite in sign for these two states. To understand this we recall that the reduced matrix element $\left\langle n l^{\prime}|| p|| n l\right\rangle=0$ for all $l$ and $l^{\prime}$ and so one can see from equation (A.1) that the direct term is always zero for all DESB states while the exchange term may be non-zero for intershell DESB states. As the DESB CI coefficients are the same for the $A=+1$ and -1 series (A.1) shows that $\left\langle\boldsymbol{p}_{1} \cdot \boldsymbol{p}_{2}\right\rangle$ is equal and opposite for the two series as billed.

3.2.3. Helium. The comparison between the intershell ${ }_{4}(2,0)_{3}^{+}$and ${ }_{4}(2,0)_{3}^{-}$rotor series of $\mathrm{He}$ is shown in table 4. Comparing the $A=+1$ and $A=-1$ series with the same $K$ and $T$, the average values of $\left\langle\cos \theta_{12}\right\rangle$ are about the same ( $\operatorname{Lin} 1983,1984,1986$, Watanabe and Lin 1986) except for states with large $L$. The Coulomb repulsion $\left\langle 1 / r_{12}\right\rangle$ is larger for the $A=+1$ states than for the $A=-1$ states, the average $\left\langle\boldsymbol{p}_{1} \cdot \boldsymbol{p}_{2}\right\rangle$ are negative for $A=+1$ states and positive for $A=-1$ states in accord with the classical molecular model. Values of $\bar{R}^{2}$ for the $A=+1$ series are larger than the $A=-1$ series. All these properties parallel those of $\mathrm{C}^{4+}$. However, important exceptions are to be 
Table 4. Tabulation of the energy and various expectation values for the two intershell ${ }_{4}(2,0)_{3}^{+}$and ${ }_{4}(2,0)_{3}^{-}$rotor series of $\mathrm{He}$.

\begin{tabular}{|c|c|c|c|c|c|c|}
\hline & $E(\mathrm{eV})$ & $\left\langle\cos \theta_{12}\right\rangle$ & $\bar{R}^{2}(\mathrm{au})^{2}$ & $\left\langle 1 / r_{12}\right\rangle(\mathrm{eV})$ & $\begin{array}{l}\left\langle\boldsymbol{p}_{1} \cdot \boldsymbol{p}_{2}\right\rangle /\left(m_{\mathrm{e}}\langle\mathrm{KE}\rangle\right) \\
\times 10^{-2}\end{array}$ & $\langle\mathrm{KE}\rangle(\mathrm{eV})$ \\
\hline \multicolumn{7}{|c|}{$(2,0)^{+}$} \\
\hline${ }^{i} S^{e}$ & -7.64 & -0.634 & 304.2 & 1.58 & -3.32 & 7.73 \\
\hline${ }^{3} \mathrm{P}^{\mathrm{O}}$ & -7.60 & -0.594 & 304.6 & 1.60 & -4.06 & 7.30 \\
\hline${ }^{1} D^{e}$ & -7.50 & -0.544 & 311.8 & 1.62 & -4.45 & 7.16 \\
\hline${ }^{3} \mathrm{~F}^{\circ}$ & -7.37 & -0.484 & 318.4 & 1.66 & -4.90 & 7.08 \\
\hline${ }^{1} G^{e}$ & -7.13 & -0.407 & 369.8 & 1.63 & -3.66 & 6.97 \\
\hline \multicolumn{7}{|c|}{$(2,0)^{-}$} \\
\hline${ }^{3} \mathrm{~S}^{\mathrm{e}}$ & -7.82 & -0.636 & 265.1 & 1.52 & 4.90 & 7.74 \\
\hline${ }^{1} \mathrm{P}^{0}$ & -7.78 & -0.603 & 263.7 & 1.54 & 5.44 & 7.70 \\
\hline${ }^{3} D^{e}$ & -7.70 & -0.539 & 261.8 & 1.59 & 6.40 & 7.60 \\
\hline${ }^{1} F^{\circ}$ & -7.56 & -0.450 & 262.5 & 1.65 & 7.33 & 7.43 \\
\hline${ }^{3} \mathrm{G}^{\mathrm{e}}$ & -7.33 & -0.346 & 276.9 & 1.70 & 6.43 & 7.20 \\
\hline
\end{tabular}

found from the behaviour of $\bar{R}^{2}$ and $\left\langle 1 / r_{12}\right\rangle$. The intershell series of He generally exhibit a molecule-like rotational expansion and a nearly constant value of the bielectronic repulsion with respect to $L$ (only the ${ }_{4}(2,0)_{3}^{-}$series shows signs of dissenting from this behaviour). This 'anomalous' behaviour is due to strong intershell mixing in the He wavefunction. Since DESB states always show rotational contraction and a rise of the bielectronic repulsion, the fact that intershell states of the He system display rotational expansion and constant interelectronic repulsion is an indication of the failure of the DESB description. This point will be further examined in section 4 .

\section{Rotational expansion and the origin of the rotational constant in helium}

\subsection{The rotational expansion}

The rotational expansion is the result of strong intershell coupling which, in turn, is due to the neglect of the screening of the outer electron by the inner electron when unscreened hydrogenic wavefunctions are used as basis functions. The lower angular momentum states involve $\mathrm{CI}$ basis functions comprised of hydrogenic orbitals of lower angular momentum. Due to the high degree of penetration of the outer electron into the inner region, the inner electron will incompletely screen the outer electron. Higher angular momentum states involve $\mathrm{Cl}$ basis functions composed of higher angular momentum orbitals so that the outer electron is less effective in penetrating the inner region. Thus the outer electron is more effectively screened, feels a lower effective charge and shifts to larger distances from the nucleus. A similar expansion of the $\mathrm{C}^{4+}$ system is more strongly inhibited by the larger energy gap between the basis states.

\subsection{The origin of the rotational constant and the 'antimolecular' behaviour of $\langle K E\rangle$}

As discussed in section 3.1, the rotor energy level spacing for the ${ }_{3}(2,0)_{3}^{+}$series of $\mathrm{C}^{4+}$ largely derives from the increase of $\left\langle 1 / r_{12}\right\rangle$. For helium, however, the strong intershell mixing with states of higher principal quantum numbers as $L$ increases, alters this such that the rotational constant derives largely from the change in the expectation 
value of the electron-nucleus potential, $\left\langle-\left(2 / r_{1}+2 / r_{2}\right)\right\rangle$. In this case, the values of $\left\langle 1 / r_{12}\right\rangle$ account for only $40 \%$ of the increase in energy up the intrashell rotor series and for most intershell states the contribution of the bielectronic repulsion to the increase in $E$ is negligible and may even go the other way for the higher intershell series.

The expectation values of the kinetic energy $\langle\mathrm{KE}\rangle$ always decreases up a rotor series, whereas the DESB values are independent of $L$ for a given rotor series. The falling value of $\langle\mathrm{KE}\rangle$ as $L$ increases up a rotor series for $\mathrm{He}$ may be viewed as an antimolecular feature; if the system were behaving in a fashion similar to a molecule $\langle\mathrm{KE}\rangle$ would show a rotor-like increase. Plots of surface charge density against $\theta_{12}$ and $\alpha=\tan ^{-1} r_{2} / r_{1}$ in figure 3 of Lin (1984) reveal that although the surface charge density is strongly peaked about some value of $\theta_{12}$ and $\alpha$, the peaks become noticeably broader as the angular momentum increases up a rotor series. The 'antimolecular' tendency for $\left\langle\cos \theta_{12}\right\rangle$ to increase for higher angular momentum is a reflection of this behaviour. Hence the falling value of $\langle\mathrm{KE}\rangle$ may be viewed as a consequence of the uncertainty principle, the volume of coordinate space available to the system for stretch and vibrational motion increases, entailing a reduction in the zero point and excitation energies of the stretch and vibrational modes. The, at best, modest rise in $\left\langle 1 / r_{12}\right\rangle$ and fall in $\langle\mathrm{KE}\rangle$ implies that the rotational constant largely derives from the term $\left\langle-\left(2 / r_{1}+\right.\right.$ $\left.\left.2 / r_{2}\right)\right\rangle$.

\section{T-doubling}

\subsection{The behaviour of the $\eta=+1$ and $\eta=-1$ series}

The $\mathrm{C}^{4+}$ intrashell ${ }_{3}(1,1)_{3}^{+}$rotor series, tabulated in table 5 , illustrates the phenomenon of $T$-doubling. $T$-doubling occurs whenever $T>0$ and refers to the near degenerate $\eta= \pm 1$ states, where $\eta=\pi(-1)^{L}$. The linear triatomic molecular model ascribes the two near degenerate states to the two degenerate vibrational bending modes. For a molecule this degeneracy is lifted by two mechanisms (Herzberg 1945): (i) the moment of inertia about the axis of molecular rotation depends on whether the bending motion is parallel or perpendicular to this axis; (ii) Coriolis forces excite the antisymmetric

Table 5. Tabulation of the energy and various expectation values for the $T$-doubled intrashell ${ }_{3}(1,1)_{3}^{+}$rotor series of $C^{4+}$ (DESB values in brackets and $\eta=\pi(-1)^{L}$ ). All intrashell DESB values of $\left(\boldsymbol{p}_{1} \cdot \boldsymbol{p}_{2}\right) / \mathrm{m}_{\mathrm{e}}$ are zero and are thus not tabulated.

\begin{tabular}{lccccc}
\hline & & & & & $\left\langle\boldsymbol{p}_{1} \cdot \boldsymbol{p}_{2}\right\rangle / m_{\mathrm{e}}$ \\
& $E(\mathrm{eV})$ & $\left\langle\cos \theta_{12}\right\rangle$ & $\bar{R}^{2}(\mathrm{au})^{2}$ & $\left\langle 1 / r_{12}\right\rangle(\mathrm{eV})$ & \\
\hline$\eta=1$ & & & & \\
${ }^{1} \mathrm{P}^{\mathrm{o}}$ & $-99.05(-98.54)$ & $-0.182(-0.148)$ & $10.94(9.75)$ & $9.27(10.30)$ & -0.11 \\
${ }^{3} \mathrm{D}^{\mathrm{e}}$ & $-98.18(-97.65)$ & $-0.062(0.0)$ & $10.35(9.25)$ & $10.09(11.20)$ & 0.054 \\
${ }^{1} \mathrm{~F}^{\mathrm{o}}$ & $-95.06(-93.67)$ & $0.097(0.222)$ & $9.92(8.5)$ & $12.36(15.18)$ & -0.122 \\
$\eta=-1$ & & & & & \\
${ }^{3} \mathrm{P}^{\mathrm{e}}$ & $-99.35(-98.98)$ & $-0.209(-0.185)$ & $10.59(9.5)$ & $9.11(9.86)$ & 0.14 \\
${ }^{1} \mathrm{D}^{\circ}$ & $-98.62(-98.25)$ & $-0.144(-0.111)$ & $9.54(8.5)$ & $9.80(10.60)$ & 0.52 \\
${ }^{3} \mathrm{~F}^{\mathrm{e}}$ & $-96.68(-96.11)$ & $-0.042(0.0)$ & $8.13(7.0)$ & $11.53(12.74)$ & 1.34 \\
\hline
\end{tabular}


stretch modes when the bending vibration takes place in a plane perpendicular to the axis of rotation.

For both of the $T$-doubled series $E$ displays molecule like rotor behaviour while $\bar{R}^{2}$ and $\left\langle\cos \theta_{12}\right.$ ) exhibit antimolecular behaviour up the rotor series. In the molecular model the intrashell ${ }_{3}(1,1)_{3}^{+}$states possess an additional quantum of vibration over the ${ }_{3}(2,0)_{3}^{+}$states and so should exhibit larger values of $\left\langle\cos \theta_{12}\right\rangle$. Examination of tables 1 and 5 confirms this behaviour. These features are well described by the DESB states, although the $\eta=+1$ states show larger intershell mixing.

The ${ }_{3}(1,1)_{3}^{+}$rotor series illustrates a trend found for all pairs of $T$-doubled states $(A= \pm 1,0)$ which have been examined in this study ( $\mathrm{He}$ as well as $\mathrm{C}^{4+}$ ), namely that the $\eta=+1$ states lie higher in energy than the $\eta=-1$ states. This result is a reversal of molecular behaviour (Herzberg 1945) and models whereby electrons are confined to concentric spheres (Ezra and Berry 1982, 1983). That intrashell states exhibit these properties has been noted before by Ezra and Berry (1982) and Watanabe and Lin (1986) who also examine the reasons for such behaviour. According to these authors $T$-doubling derives from the behaviour of the wavefunction at $\theta_{12}=0$ which in turn influences the value of the bielectronic repulsion. One notes that this mechanism has little to do with the mechanisms which drive $l$-doubling in linear molecules, although the analysis remains to be extended to intershell states. Examination of the data for intrashell states (table 5) and intershell states (not shown in the tables) indeed shows that the bielectronic repulsion entirely accounts for the splitting between $\eta=+1$ and -1 DESB states. However, the bielectronic repulsion often accounts for no more than $50 \%$ of the splitting for the $\mathrm{C}^{4+}$ system.

From table 5 it may be seen that the $\eta=+1$ series of $\mathrm{C}^{4+}$ shows a less negative $\left\langle\cos \theta_{12}\right.$ ) and a larger $\bar{R}^{2}$ than the $\eta=-1$ series, properties which also feature in the DESB approximation.

The He system (no tables) again always exhibits a smaller value of $\bar{R}^{2}$ for the $\eta=-1 A= \pm 1$ series. However, if one compares the two $\eta= \pm 1$ series and looks at $\left\langle\cos \theta_{12}\right\rangle$ and $\left\langle 1 / r_{12}\right\rangle$, the trends displayed by the $\mathrm{C}^{4+}$ system (see above) become less clear and non-existent respectively for the He system. In fact, $\left\langle 1 / r_{12}\right\rangle$ is usually larger for the $\eta=-1$ series as is $\langle\mathrm{KE}\rangle$. This means that the difference in $\left\langle-2 / r_{1}-2 / r_{2}\right\rangle$ between the two $T$-doubled series more than compensates for the higher values of $\left\langle 1 / r_{12}\right\rangle$ and $\langle\mathrm{KE}\rangle$ for the $\eta=-1$ series, driving the $\eta=+1$ series higher than the $\eta=-1$ series.

\subsection{Behaviour of $\left\langle\boldsymbol{p}_{1} \cdot \boldsymbol{p}_{2}\right\rangle$}

For those $\mathrm{C}^{4+}$ intrashell ${ }_{3}(1,1)_{3}^{+}$states for which $\left\langle\boldsymbol{p}_{1} \cdot \boldsymbol{p}_{2}\right\rangle$ is sufficiently large to reliably determine the sign (the ${ }^{1} \mathrm{D}^{\mathrm{o}}$ and ${ }^{3} \mathrm{~F}^{\mathrm{e}} \eta=-1$ states), $\left\langle\boldsymbol{p}_{1} \cdot \boldsymbol{p}_{2}\right\rangle$ is positive. This is in agreement with the classical model as one would expect a positive value for $\left\langle\boldsymbol{p}_{1} \cdot \boldsymbol{p}_{2}\right\rangle$ for a system with vibrational angular momentum, although the results for the other four states are not accurate enough to draw any definitive conclusions.

In the molecular picture, the intershell $(1,1)_{3}^{ \pm}$series (not tabulated) superimpose stretch modes upon the mode with one unit of vibrational angular momentum. According to the classical model the antisymmetric stretch modes of the intershell $(1,1)_{3}^{-}$ series have a positive contribution to $\left\langle\boldsymbol{p}_{1} \cdot \boldsymbol{p}_{2}\right\rangle$ leaving it positive overall and indeed these intershell series do display positive values for $\left\langle\boldsymbol{p}_{1} \cdot \boldsymbol{p}_{2}\right\rangle$. For the $(1,1)_{3}^{+}$series the symmetric stretch modes should have a negative contribution to $\left\langle\boldsymbol{p}_{1} \cdot \boldsymbol{p}_{2}\right\rangle$ and indeed $\left\langle\boldsymbol{p}_{1} \cdot \boldsymbol{p}_{2}\right\rangle$ does become negative for all the $\eta=-1 \mathrm{C}^{4+}$ intershell series of this study. Unfortunately the present method does not provide sufficiently accurate results for the 
$A=+1, \eta=+1$ series to reliably determine the sign of $\left\langle\boldsymbol{p}_{1} \cdot \boldsymbol{p}_{2}\right\rangle$. The $(1,1)_{3}^{ \pm}$series of $\mathrm{He}$ also behave in a similar fashion.

\section{Higher 'vibrationally' excited states: the $(0,0)_{3}^{+}$rotor series}

The energy and other expectation values for the intrashell ${ }_{3}(0,0)_{3}^{+}$series of $\mathrm{C}^{4+}$ are shown in table 6. Again $E$ shows molecular behaviour while $\bar{R}^{2}$ and $\left\langle\cos \theta_{12}\right\rangle$ display antimolecular properties along the rotor series. According to the molecular model the states of this series are excited with two units of vibrational quanta. In keeping this picture the intrashell ${ }_{3}(0,0)_{3}^{+}$series displays larger values for $\left\langle\cos \theta_{12}\right\rangle$ than the ${ }_{3}(1,1)_{3}^{+}$ series with one quanta of vibrational energy, and, in fact, these are positive for every member of the series. This means that the two electrons have a greater probability of being on the same side of the nucleus. One notes that the last member of this series expands over the previous member, although this is the only example of this behaviour in the $A= \pm 1 \mathrm{C}^{4+}$ series of this study.

Table 6. Tabulation of the energy and various expectation values for the intrashell ${ }_{3}(0,0)_{3}^{+}$ rotor series of $\mathrm{C}^{4+}$ (DESB values in brackets). All intrashell DESB values of $\left\langle\boldsymbol{p}_{1} \cdot \boldsymbol{p}_{2}\right\rangle / m_{\mathrm{e}}$ are zero and are thus not tabulated.

\begin{tabular}{llllll}
\hline & $E(\mathrm{eV})$ & $\left\langle\cos \theta_{12}\right\rangle$ & $\bar{R}^{2}(\mathrm{au})^{2}$ & $\left\langle 1 / \boldsymbol{r}_{12}\right\rangle(\mathrm{eV})$ & $\begin{array}{l}\left\langle\boldsymbol{p}_{1} \cdot \boldsymbol{p}_{2}\right\rangle / m_{\mathrm{e}} \\
(\mathrm{eV})\end{array}$ \\
\hline$(0,0)^{+}$ & & & & & \\
${ }^{1} \mathrm{~S}^{\mathrm{e}}$ & $-96.89(96.04)$ & $0.032(0.111)$ & $10.52(9.25)$ & $11.11(12.81)$ & 3.92 \\
${ }^{3} \mathrm{P}^{\mathrm{o}}$ & $-96.53(95.88)$ & $0.104(0.167)$ & $10.08(8.875)$ & $11.57(12.97)$ & 5.51 \\
${ }^{1} \mathrm{D}^{\mathbf{e}}$ & $-93.41(81.25)$ & $0.227(0.277)$ & $10.20(8.125)$ & $13.72(16.82)$ & 6.65 \\
\hline
\end{tabular}

For intrashell states the DESB would predict zero values of $\left\langle\boldsymbol{p}_{1} \cdot \boldsymbol{p}_{2}\right\rangle$. The large values of $\left\langle\boldsymbol{p}_{1} \cdot \boldsymbol{p}_{2}\right\rangle$ indicates that intershell mixing is quite large. As $A=+1$ for this group, $\left\langle\boldsymbol{p}_{1} \cdot \boldsymbol{p}_{2}\right\rangle$ is expected to be positive and indeed the calculated values in table 6 are consistent with this (classical) picture.

\section{7. $A=0$ doubly excited states}

\subsection{Values of $\left\langle\boldsymbol{p}_{1} \cdot \boldsymbol{p}_{2}\right\rangle$}

The energies and values for $\left\langle\boldsymbol{p}_{1} \cdot \boldsymbol{p}_{2}\right\rangle$ of the $A=0$ states of the $\mathrm{C}^{4+} 3 l 4 l^{\prime}$ manifold are given in table 7 . Values of $\left\langle\boldsymbol{p}_{1} \cdot \boldsymbol{p}_{2}\right\rangle$ from the full TDM calculation are of opposite sign for states with different $S$, a property which derives from the DESB states since, as explained in section 3.2.2), the DESB $\left\langle\boldsymbol{p}_{1} \cdot \boldsymbol{p}_{2}\right\rangle$ values are equal and opposite for states of differing $S$. The full TDM calculation also shows that the dependence of the sign of $\left\langle p_{1} \cdot p_{2}\right\rangle$ on the spin $S$ is reversed for the $T$-doubled $\eta=-1$ states as compared with the $\eta=+1$ states, and this behaviour too is inherent in the DESB state expectation values.

For helium the pair of $A=0$ singlet and triplet states also tend to display opposite signs for $\left\langle\boldsymbol{p}_{1} \cdot \boldsymbol{p}_{2}\right\rangle$, although we have witnessed a few exceptions for which the values of $\left\langle\boldsymbol{p}_{1} \cdot \boldsymbol{p}_{2}\right\rangle$ should be large enough to be above the error inherent in the calculation. 
Table 7. Tabulation of $E$ and $\left\langle p_{1} \cdot p_{2}\right\rangle / m_{\mathrm{e}}$ for lowest $A=0$ intershell states of $\mathrm{C}^{4+}$ (DESB values in brackets and $\eta=\pi(-1)^{L}$ ).

\begin{tabular}{|c|c|c|c|c|c|}
\hline & \multicolumn{2}{|c|}{$\eta=+1$} & & \multicolumn{2}{|c|}{$\eta=-1$} \\
\hline & $E(\mathrm{eV})$ & $\left\langle\boldsymbol{p}_{1} \cdot \boldsymbol{p}_{2}\right\rangle / m_{\mathrm{e}}(\mathrm{eV})$ & & $E(\mathrm{eV})$ & $\left\langle\boldsymbol{p}_{1} \cdot \boldsymbol{p}_{2}\right\rangle / m_{\mathrm{e}}(\mathrm{eV})$ \\
\hline \multicolumn{6}{|l|}{${ }_{4}(-2,0)_{3}^{0}$} \\
\hline & $-73.91(-72.57)$ & $4.38(4.02)$ & & & \\
\hline${ }^{3} \mathrm{P}^{\circ}$ & $-74.60(-73.80)$ & $-0.20(-4.02)$ & & & \\
\hline \multicolumn{6}{|l|}{${ }_{4}(-1,1)_{3}^{0}$} \\
\hline${ }^{1} D^{e}$ & $-74.41(-72.75)$ & $4.88(7.88)$ & ${ }^{3} \mathrm{D}^{\circ}$ & $-75.44(-74.77)$ & $6.04(5.92)$ \\
\hline${ }^{3} D^{e}$ & $-75.40(-74.71)$ & $-3.71(-7.88)$ & ${ }^{1} \mathrm{D}^{e}$ & $-76.12(-75.66)$ & $-2.72(-5.92)$ \\
\hline \multicolumn{6}{|l|}{${ }_{4}(0,0)_{3}^{0}$} \\
\hline & $-74.06(-73.11)$ & $5.37(12.11)$ & & & \\
\hline${ }^{3} \mathrm{~F}^{\circ}$ & $-75.79(-75.52)$ & $-7.31(-12.11)$ & & & \\
\hline \multicolumn{6}{|l|}{${ }_{4}(0,2)_{3}^{0}$} \\
\hline${ }^{1} \mathrm{~F}^{\circ}$ & $\begin{array}{l}-75.74(-75.26) \\
-77.13(-76.83)\end{array}$ & $\begin{array}{c}3.52(10.06) \\
-2.43(-10.06)\end{array}$ & ${ }^{3} \mathrm{~F}^{\mathrm{e}}$ & $\begin{array}{l}-76.34(-75.71) \\
-77.16(-76.88)\end{array}$ & $\begin{array}{c}7.54(10.09) \\
-4.57(-10.09)\end{array}$ \\
\hline \multicolumn{6}{|l|}{${ }_{4}(1,1)_{3}^{0}$} \\
\hline${ }^{1} G^{e}$ & $-75.06(-73.22)$ & $9.52(18.17)$ & & & \\
\hline${ }^{3} \mathrm{G}^{\mathrm{e}}$ & $-76.79(-76.31)$ & $-12.78(-18.17)$ & & & \\
\hline
\end{tabular}

However, we cannot completely dismiss the possibility that these unusual values may be spurious. We note that accurate calculations of the singly excited $P$ and $D$ levels of helium produce values of $\left\langle\boldsymbol{p}_{1} \cdot \boldsymbol{p}_{2}\right\rangle$ which are of opposite sign for the singlet and triplet states (Kono and Hattori 1986).

\subsection{Energy ordering between singlet and triplet states}

Table 7 also gives the energies of the pair of singlet and triplet states. For $\eta=+1$, as noted earlier (Lin 1984 and section 5 of this paper), the energy of the triplet state is always lower than the singlet. This is not the case for $\eta=-1$ states, where the energy of the singlet state is lower than the triplet state for the pair, as illustrated in table 7.

\section{Summary and conclusions}

This article examines a number of expectation values of intrashell and intershell doubly excited states of the $\mathrm{C}^{4+}$ and $\mathrm{He}$ systems where the wavefunctions are generated in two different levels of approximation by the 'accurate' truncated diagonalisation method and approximated by a single DESB function. The systematics of these expectation values have been related to the linear triatomic molecular model of doubly excited states (Herrick 1983, Berry 1989).

Five expectation values, $\left\langle\cos \theta_{12}\right\rangle,\langle\mathrm{KE}\rangle=\frac{1}{2}\left\langle\boldsymbol{p}_{1}^{2}+\boldsymbol{p}_{2}^{2}\right\rangle,\left\langle\boldsymbol{p}_{1} \cdot \boldsymbol{p}_{2}\right\rangle,\left\langle 1 / r_{12}\right\rangle$ and $\bar{R}^{2}=\left\langle\boldsymbol{R}^{2}\right\rangle=$ $\left\langle r_{1}^{2}+r_{2}^{2}\right\rangle$, in addition to the resonance energy $E$ are studied for the $N=3$ Rydberg series of $\mathrm{C}^{4+}$ and the $N=2$ and 3 Rydberg series of He. If doubly excited states were genuinely molecular then as $L$ increases $E$ would display a rotor structure, $\langle\mathrm{KE}\rangle$ and $\left\langle\cos \theta_{12}\right\rangle$ would increase and decrease respectively while $\bar{R}^{2}$ would become larger 
(rotational expansion). The behaviour of $\left\langle\boldsymbol{p}_{1} \cdot \boldsymbol{p}_{2}\right\rangle$ within the molecular model has been discussed in the introduction. When one examines the actual behaviour of these expectation values one finds that for both the $\mathrm{C}^{4+}$ and He systems the energy displays an approximate (but truncated) rotor series, while the behaviour of the momentum correlation $\left\langle\boldsymbol{p}_{1} \cdot \boldsymbol{p}_{2}\right\rangle$ is found to be in good agreement with the classical molecular model of Krause et al (1987) for the large number of states of this study. Both molecular behaviours are predicted by the DESB functions as well as by the TDM calculations, except that DESB functions predict zero values of $\left\langle\boldsymbol{p}_{1} \cdot \boldsymbol{p}_{2}\right\rangle$ for intrashell states.

The doubly excited states studied also display many antimolecular features: (i) the expectation value of $\cos \theta_{12}$ rises along the rotor series; (ii) $\langle\mathrm{KE}\rangle$ decreases along the rotor series; (iii) as measured by $\bar{R}^{2}$, the states contract along the rotor series (except for the intershell series of helium). Although a single DESB function provides a good approximation to a state of the $\mathrm{C}^{4+}$ system, the same cannot be said of the He. In particular, for the intershell series of He the intershell coupling has sufficient strength to produce a rotational expansion of the system reminiscent of a molecule.

There are a number of properties of doubly excited states which resemble those observed in molecules but are of different physical origins.

(i) The rotor structure does not derive from the inertia, but comes instead from the potential energies. For $C^{4+}$ and DESB states the rotational constant $B$ originates from the bielectronic repulsion, however the He system exhibits a large amount of intershell mixing and this brings about a change in the origin of $B$. For $\mathrm{He}$ the rotor structure derives from the electron-nucleus potential $\left\langle-\left(2 / r_{1}+2 / r_{2}\right)\right\rangle$ since $\left\langle 1 / r_{12}\right\rangle$ is flat and $\langle\mathrm{KE}\rangle=\left\langle\boldsymbol{p}_{1}^{2}+\boldsymbol{p}_{2}^{2}\right\rangle$ falls with $L$. This antimolecular fall of $\langle\mathrm{KE}\rangle$ with $L$ is interpreted to be a consequence of the uncertainty principle where the volume available for the system to execute stretch and vibration motions increases with $L$. The antimolecular behaviour of $\left\langle\cos \theta_{12}\right\rangle$ is seen to be an aspect of this increasing volume.

(ii) When $T \neq 0 T$-doubling occurs. It is found that the $\eta=+1$ states always lie higher in energy than the $\eta=-1$ states independent of $A$ or the system ( $\mathrm{He}$ or $\mathrm{C}^{4+}$ ). This is a reversal of molecular behaviour (Herzberg 1945) and models in which electrons are confined to concentric spheres (Ezra and Berry 1982, 1983). For intrashell states of two-electron atoms, Ezra and Berry (1982) and Watanabe and Lin (1986) have proposed that $T$-doubling is due to the behaviour of the wavefunction near $\theta_{12}=0$ which is reflected in the bielectronic repulsion. One notes that this mechanism is different from the mechanisms driving the analogous $l$-doubling in molecules where the degeneracy of the two vibrational modes is lifted by the Coriolis interaction and the differing moments of inertia about the axis of rotation of the two vibrational modes (Herzberg 1945). In the DESB approximation the bielectronic repulsion entirely accounts for the splitting, but for the $\mathrm{C}^{4+}$ system it often accounts for no more than $50 \%$ of the total splitting. For He, however, the differences in $\left\langle 1 / r_{12}\right\rangle$ and $\langle\mathrm{KE}\rangle$ between the two series usually go in the opposite direction to the energy splitting. It is the difference in electron-nucleus potential which drives the $\eta=+1$ higher than the $\eta=-1$ states for this system.

\section{Acknowledgment}

This work is supported in part by the US Department of Energy, Office of Basic Energy Research, Division of Chemical Sciences. We also wish to thank Xin Yu Zhang for preparing many of the tables. 


\section{Appendix}

This appendix outlines the calculation of the matrix elements of $\left\langle\boldsymbol{p}_{1} \cdot \boldsymbol{p}_{2}\right\rangle$ between the $\mathrm{Cl}$ basis functions.

The symmetric or antisymmetric spatial basis functions are given by

$$
\left|n_{1} l_{1}, n_{2} l_{2} ; L M\right\rangle=\mathbf{P}\left\{\sum_{m_{1} m_{2}}\left(l_{1} m_{1} l_{2} m_{2} \mid L M\right)\left|n_{1} l_{1}\right\rangle\left|n_{2} l_{2}\right\rangle\right\}
$$

where $\left|n_{i} l_{i}\right\rangle$ are the one particle basis functions with angular momentum $l_{i}$ and $\mathbf{P}$ is the symmetrisation operator (for singlet states) or antisymmetrisation operator (for triplet states). One can then write the matrix element of $\boldsymbol{p}_{1} \cdot \boldsymbol{p}_{2}$ as follows

$$
\begin{aligned}
&\left\langle n_{1}^{\prime} l_{1}^{\prime}, n_{2}^{\prime} l_{2}^{\prime} ; L^{\prime} M^{\prime}\left|\boldsymbol{p}_{1} \cdot \boldsymbol{p}_{2}\right| n_{1} l_{1}, n_{2} l_{2} ; L M\right\rangle \\
&=(-1)^{l_{1}+l_{2}^{\prime}} \delta_{L L^{\prime}} \delta_{M M^{\prime}}\left[(-1)^{L}\left\{\begin{array}{ccc}
L & l_{2}^{\prime} & l_{1}^{\prime} \\
1 & l_{1} & l_{2}
\end{array}\right\}\left\langle n_{1}^{\prime} l_{1}^{\prime}|| \boldsymbol{p}_{1}|| n_{1} l_{1}\right\rangle\left\langle n_{2}^{\prime} l_{2}^{\prime}|| \boldsymbol{p}_{2}|| n_{2} l_{2}\right\rangle\right. \\
&\left.+(-1)^{S}\left\{\begin{array}{ccc}
L & l_{2}^{\prime} & l_{1}^{\prime} \\
1 & l_{2} & l_{1}
\end{array}\right\}\left\langle n_{1}^{\prime} l_{1}^{\prime}|| \boldsymbol{p}_{1}|| n_{2} l_{2}\right\rangle\left\langle n_{2}^{\prime} l_{2}^{\prime}|| \boldsymbol{p}_{2}|| n_{1} l_{1}\right\rangle\right] .
\end{aligned}
$$

The first term is the direct term and the second is the exchange term, $S$ is the total spin, $\left\langle n^{\prime} l^{\prime}|| \boldsymbol{p}|| n l\right\rangle$ is the reduced matrix element of the momentum $\boldsymbol{p}=-\mathrm{i} \nabla$ and is given by

$$
\left\langle n^{\prime} l^{\prime}||-\mathrm{i} \nabla|| n l\right\rangle=\delta_{l^{\prime}(l+1)} H_{n^{\prime} n l}-\delta_{l^{\prime}(l-1)} H_{n n^{\prime}(l-1)}
$$

where

$$
H_{n^{\prime} n l}=\sqrt{(l+1)} \int_{0}^{\infty} F_{n^{\prime}(l+1)}(r)\left\{\frac{\partial}{\partial r}-\frac{l}{r}\right\} F_{n l}(r) r^{2} \mathrm{~d} r
$$

and $F_{n l}(r)$ is the radial basis function. This may be obtained using the Racah methods described in Condon and Odabasi (1980).

If $\left|n_{1} l_{1}, n_{2} l_{2} ; L M\right\rangle$ is formed from hydrogenic functions, the derivative in the integrand of $H_{n^{\prime} n l}$ may be eliminated by using the bound state $S O(4)$ algebra related recursion relations (Chattarji 1967)

$$
\begin{aligned}
& {\left[\lambda l\left(\frac{\partial}{\partial r}+\frac{(l+1)}{r}\right)-1\right] F_{n l}(r)=\left(1-\frac{l^{2}}{n^{2}}\right)^{1 / 2} F_{n(l-1)}(r)} \\
& {\left[\lambda(l+1)\left(\frac{\partial}{\partial r}-\frac{l}{r}\right)+1\right] F_{n l}(r)=\left(1-\frac{(l+1)^{2}}{n^{2}}\right)^{1 / 2} F_{n(l+1)}(r)}
\end{aligned}
$$

where the phase convention is such that the $F_{n l}(r)$ are all of the same sign near the origin and $\lambda=1 / Z$ is the first Bohr radius of the system.

With the recursion relations one finds that

$$
\left\langle n^{\prime} l^{\prime}||-\mathrm{i} \nabla|| n l\right\rangle=-\delta_{l^{\prime}(l+1)} I_{n^{\prime} n l}-\delta_{l^{\prime(l-1)}} I_{n n^{\prime}(l-1)}
$$

where

$$
I_{n^{\prime} n l}=\frac{1}{\lambda \sqrt{(l+1)}}\left[G_{n^{\prime} n l}+\delta_{n n^{\prime}}\left(1-\frac{(l+1)^{2}}{n^{2}}\right)^{1 / 2}\right]
$$


and

$$
G_{n^{\prime} n l}=\int_{0}^{\infty} F_{n^{\prime}(l+1)}(r) F_{n l}(r) r^{2} \mathrm{~d} r .
$$

$G_{n^{\prime} n l}$ is then evaluated numerically.

\section{References}

Berry R S 1989 Contemp. Phys. 301 and references therein

Berry R S and Krause J L 1988 Adv. Chem. Phys. 7035 and references therein

Chattarji D 1967 Nuovo Cimento A 48524

Chen Z and Lin C D 1990 Phys. Rev. A to be published

Condon E U and Odabaşi H 1980 Atomic Structure (Cambridge: Cambridge University Press) pp 235-42

Cooper J W, Fano U and Prats F 1963 Phys. Rev. Lett. 10518

Ezra and Berry 1982 Phys. Rev. A 251513

1983 Phys. Rev. A 281989

Feagin J M 1987 Fundamental Processes of Atomic Dynamics ed J S Briggs, H Kleinpoppen and H O Lutz (New York: Plenum) pp 275-300 and references therein

Feagin J M and Briggs J S 1987 Phys. Rev. Lett. 57984

- 1988 Phys. Rev. A 374599

Herrick D R 1983 Adv. Chem. Phys. 521

Herrick D R and Keliman M E 1980 Phys. Rev. A 21418

Herrick D R, Kellman M E and Poliak R D 1980 Phys. Rev. A 221517

Herrick D R and Sinanoğlu O 1975 Phys. Rev. A 1197

Herschbach D R, Loeser J G and Watson D K 1988 Z. Phys. D 10195

Herzberg G 1945 Molecular Spectra and Molecular Structure (New York: Van Nostrand) Vol II

Ho Y K 1983 Phys. Rep. 991 and references therein

— 1987 Phys. Rev. A 352035

1989 Z. Phys. D 11277

Iwai M and Nakamura H 1989a Phys. Rev. A 402247 1989b Phys. Rev. A 406695

Junker B R 1982 Adv. At. Mol. Phys. 18207 and references therein

Kellman M E and Herrick D R 1980 Phys. Rev. A 221536

Kono A and Hattori S 1986 Phys. Rev. A 341727

Krause J L, Morgan J D III and Berry R S 1987 Phys. Rev. A 353189

Lin C D 1983 Phys. Rev. Lett. 511348

1984 Phys. Rev. A 291019

1986 Adv. At. Mol. Phys. 22 77-142 and references therein

Lipsky L, Anania R and Corneely M J 1977 At. Data Nucl. Data Tables 20727 and references therein

Macek J H 1968 J. Phys. B: At. Mol. Phys. 1831

Madden R P and Codling K 1963 Phys. Rev. Lett. 10516

Mølmer K and Taulbjerg K 1988 J. Phys. B: At. Mol. Opt. Phys. 211739

Reinhardt W P 1982 Ann. Rev. Phys. Chem. 33233 and references therein

Sinanoǧlu O and Herrick D R 1975a J. Chem. Phys. 62886 1975b Phys. Lett. 31373

Watanabe S and Lin C D 1986 Phys. Rev. A 34823

Wulfman C 1973 Chem. Phys. Lett. 23370 